Research Paper

\title{
Effects of one-lung flooding on porcine haemodynamics and gas exchange
}

\author{
Thomas Lesser $^{\circledR}$, Frank Wolfram¹, Conny Braun², Reiner Gottschall ${ }^{3}$ \\ 1. Department Thoracic and Vascular Surgery, SRH Wald-Klinikum Gera, Teaching Hospital of Jena University Hospital, Strasse des Friedens 122 , Gera \\ D-07548, Germany. \\ 2. Central Experimental Animal Facility, University Hospital Jena, Location Dornburger Strasse 23a, Jena D-07743, Germany. \\ 3. Doctor Emeritus, Department of Anaesthesiology and Intensive Care, Jena University Hospital, Am Klinikum 1, Jena D-07747, Germany.
}

$\triangle$ Corresponding author: Department of Thoracic and Vascular Surgery, SRH Wald-Klinikum Gera, Teaching Hospital of Jena University Hospital, Strasse des Friedens 122, Gera D-07548, Germany. E-mail address: thomas.lesser@wkg.srh.de; Phone: 0049 3658283151; Fax: 00493659293159.

(0) The author(s). This is an open access article distributed under the terms of the Creative Commons Attribution License (https://creativecommons.org/licenses/by/4.0/). See http://ivyspring.com/terms for full terms and conditions.

Received: 2020.07.20; Accepted: 2020.10.07; Published: 2020.10.23

\begin{abstract}
Background and aim: We established a porcine model of one-lung flooding (OLF) that can be used for research on the use of ultrasound for lung tumour detection, ultrasound-guided transthoracic needle biopsy, and tumour ablation. However, OLF requires one-lung ventilation (OLV) and eliminates the recruitment strategies of the nonventilated lung. During thoracic surgery, OLV alone can be associated with hypoxia, hypercapnia, and right ventricular overload. Here, we examined whether OLF influences haemodynamics and gas exchange indices during and after OLV/OLF compared with OLV/apnoea and two-lung ventilation (TLV) following deflooding.

Methods: Fourteen pigs were included in this study: five were allocated to the control group (CO) and nine were assigned to the OLF group (OLF). Assessments of haemodynamics, gas exchange, and lung sonography were performed after baseline measurements, during OLV/apnoea, OLV/OLF, and after deflooding and TLV. The volume of extravascular lung water was also measured.

Results: OLF induced no significant deterioration of oxygenation or ventilation during OLF or after deflooding and TLV. Color-coded duplex sonography of the pulmonary artery in the flooded lung demonstrated an oscillating flow that corresponded to intrapulmonary circulatory arrest. After flooding of the nonventilated lung, the partial pressure of $\mathrm{O}_{2}$ in the arterial blood increased and the shunt fraction decreased significantly compared to OLV/apnoea conditions. After deflooding and TLV, haemodynamics and gas exchange indices showed no differences compared to the $\mathrm{CO}$ group and baseline values, respectively.

Conclusions: OLF is safe to use during acute animal experimentation. No clinically relevant deterioration of haemodynamics or gas exchange occurred during or after OLF. Due to the circulatory arrest in the flooded lung, the right-to-left shunt volume in the nonventilated lung was minimized. Survival experiments are necessary to further assess the utility of this method.
\end{abstract}

Key words: orcine model of one-lung flooding, haemodynamics, gas exchange

\section{Introduction}

One-lung flooding (OLF) was developed to enable complete lung sonography of the flooded lung $[1,2]$. This novel method provides new diagnostic and therapeutic options for clarifying small pulmonary nodules and for performing focused ultrasound ablation of lung tumours. Thus, it could make the diagnosis and treatment of human lung tumours more efficient and less invasive [3-5]. OLF requires one-lung ventilation (OLV) and eliminates the recruitment strategies of the nonventilated lung. OLV alone (apnoea of the nonventilated lung, OLV/ apnoea) is associated with many problems such as hypoxia, hypercapnia or right ventricular overload [6-8]. Many recruitment strategies, such as the application of continuous positive airway pressure (CPAP), high-frequency jet ventilation (HFJV), and intermitted manual ventilation of the nonventilated lung, are commonly used during thoracic surgery to avoid threatening disturbances in gas exchange when using OLV alone [9-13]. These strategies are 
impossible to implement during OLV/OLF.

The effects of OLV/OLF on haemodynamics and gas exchange with closed thorax have not been studied to date. OLF after atelectasis of the nonventilated lung following thoracotomy or thoracoscopy has a positive influence on oxygenation and shunt fraction [14,15]. However, the chest opening should be avoided in future clinical use because ultrasound-guided interventions such as focused ultrasound ablation of lung tumours are possible transcutaneously. In addition, later studies showed that atelectasis of the lung to be flooded is also not necessary [2]. Only a few studies of gas exchange during whole-lung lavage exist, which describe a therapeutic method to physically remove proteinaceous materials from the alveolar space. The highest level of oxygen saturation is usually seen during the completion of the filling phase, whereas the oxygen level drops as the lavaged lung is emptied [16-18].

Prior to the implementation of OLF on humans, the safety of the method must be studied. Pigs are commonly used as animal models of the human respiratory and cardiovascular systems because of their comparable physiology to that of humans [19-21]. Many porcine experimental studies have investigated haemodynamics, oxygenation, and pulmonary shunt fraction and perfusion during OLV [22-24]. In this study, we examined whether OLF influences the haemodynamics and gas exchange indices during and after OLV/OLF compared with OLV/apnoea and two-lung ventilation (TLV).

\section{Materials and Methods}

After approval provided by the Veterinary Department of the Thuringian State Authority for Food Protection and Fair Trading (TLLV Reg. 22-2684-04-WKG-16-002), this study was performed in laboratories at the Central Experimental Animal Facility, University Hospital Jena. All animals were kept in groups and housed for 4 days prior to the study and were thus acclimated to their new surroundings. All procedures were performed in compliance with the National Animal Protection Act.

\section{Animal preparation}

Fourteen juvenile female pigs (German Landrace, mean age 12.4 weeks), weighing an average of $37 \mathrm{~kg}$ (range: $35-40 \mathrm{~kg}$ ), were included in this study. Before the experiments began, the health status of each animal was assessed by a veterinarian.

Ketamine $(25 \mathrm{mg} / \mathrm{kg})$ and midazolam $(0.2$ $\mathrm{mg} / \mathrm{kg}$ ) were administered intramuscularly as premedicants to each pig. General anaesthesia was induced by the administration of propofol $(3 \mathrm{mg} / \mathrm{kg})$ and fentanyl $(2.7 \mu \mathrm{g} / \mathrm{kg})$ via a peripheral vein and maintained with a continuous infusion of propofol $(6$ $\mathrm{mg} / \mathrm{kg} / \mathrm{h})$ and fentanyl $(2.7 \mu \mathrm{g} / \mathrm{kg})$ every hour. After the onset of anaesthesia, the pigs were intubated transorally using a single lumen endotracheal tube (6.5 ID, Dahlhausen, Köln, Germany). Pancuronium bromide, $(0.06 \mathrm{mg} / \mathrm{kg}$, IV) was administered every hour as a muscle relaxant, and mechanical ventilation was performed using an ICU ventilator (Servo $900 \mathrm{C}$, Siemens AG, Munich, Germany) with pressurecontrolled settings [fraction of inspired oxygen $\left(\mathrm{FiO}_{2}\right)$ : 0.4 ; target tidal volume: $10 \mathrm{~mL} / \mathrm{kg}$; inspiratory to expiratory ratio: 1:1.9; respiratory rate: 20 breaths/ min; and positive end-expiratory pressure: $4 \mathrm{~cm} \mathrm{H}_{2} \mathrm{O}$ ]. These ventilator settings were maintained throughout the experiment (optimized based on an end-tidal $\mathrm{CO}_{2}$ range of 35-45 mmHg), except for $\mathrm{FiO}_{2}$, which was increased to 1.0 at the time of blood sample collection for blood gas analysis (to determine the pulmonary shunt fraction) and for denitrogenating of the lung before OLF.

A sterile technique was used to place an arterial catheter (Arterial Leader Cath 2.7 Fr; Vygon, Ecouen, France), which was advanced $10 \mathrm{~cm}$ into position in the central common carotid artery for haemodynamic monitoring and arterial blood gas sampling. A flow-directed pulmonary artery catheter (6 Fr, Swan Ganz, Edwards Lifesciences, Irvine, CA, USA) was inserted through an 8.0 Fr introducer sheath (Arrow International, Reading, PA, USA) that was placed into the right internal jugular vein. Then, a left-sided, double lumen endobronchial tube ( $35 \mathrm{Fr}$, DLT), which was designed for use in pigs and specifically made for this study (Medicoplast, International $\mathrm{GmbH}$, Illingen, Germany), was placed with the pig in the supine position via an airway exchange catheter (11.0 Fr, $100 \mathrm{~cm}$, extra-firm with a soft tip, COOK Deutschland $\mathrm{GmbH}$, Mönchengladbach, Germany). The correct position of the tube was confirmed by fibreoptic bronchoscopy (BF 3C30, Olympus, Tokyo, Japan). A cuff controller (VBM Medizintechnik $\mathrm{GmbH}$, Sulz, Germany) was used to maintain constant cuff pressures $\left(50 \mathrm{~cm} \quad \mathrm{H}_{2} \mathrm{O}\right.$ for both endobronchial and tracheal cuffs).

All animals were connected to a Datex monitor (Datex AS3 Monitoring System, Datex-Ohmeda Corp., Helsinki, Finland) for continuous vital data assessment (haemodynamic and respiratory parameters, including intrapulmonary pressure). All blood gas measurements were analysed using a blood gas analyser (Rapidpoint 405, Siemens Healthcare, Erlangen, Germany), and the output was used to calculate the pulmonary shunt fraction. 


\section{Experimental protocol}

The experimental (OLF) and control (CO) groups were studied successively. After initial preparations, baseline measurements were performed following a 30-minute period of stabilisation under TLV with the animals in the supine position. Thereafter, the left bronchial lumen of the DLT was disconnected from the ventilator to perform one-lung ventilation with apnoea of the nonventilated lung (OLV/apnoea) for 15 minutes under a $\mathrm{FiO}_{2}$ of 1.0. Then, both lungs were continuously ventilated with a $\mathrm{FiO}_{2}$ of 1.0 for 20 minutes to enable the denitrogenation of the lung as a requirement for complete OLF.

The animals were placed in the left lateral decubitus position (the lung to be flooded down), and OLF performed as follows: The left bronchial lumen of the DLT was disconnected from the ventilator, and the infusion system was immediately connected to the left tube leg. The left lung was slowly filled (single filling) with degassed and warmed $\left(37^{\circ} \mathrm{C}\right)$ isotonic saline that flowed passively from an infusion bottle suspended $50 \mathrm{~cm}$ above heart level. The volume to be infused was estimated as one-half of the functional residual capacity of the lung $(12.5 \mathrm{~mL} / \mathrm{kg})$. To prevent barotrauma and bubble formation, the saline volume was infused no faster than $125 \mathrm{~mL} / \mathrm{min}$ (assuming a basal oxygen consumption of $250 \mathrm{~mL} / \mathrm{min}$ [25]). Complete saline filling was monitored using transcutaneous lung ultrasound. At the end of the filling process, the animals were returned to the supine position, and an intrapulmonary catheter (Fogarty, Tru-lumen Embolectomy Catheter, 5F, Edwards Lifesciences, Unterschleissheim, Germany) was introduced via the left bronchial lumen of the DLT using a multiport airway adapter to avoid fluid outflow (Arndt Endobronchial Blocker Set, Cook Medical Europe, Ireland). The position of the pulmonary artery catheter and the intrapulmonary catheter were documented using ultrasound and chest X-ray analyses (Fig. 1).

The status of OLV/OLF was maintained for 3 hours. Thereafter, the fluid was passively drained from the left lung by placing the animal in the Trendelenburg position followed by TLV over a 30 -minute period (15 minutes in the right lateral position and 15 minutes in the supine position). The volume of the recovered fluid was then measured. In the $\mathrm{CO}$ group, after baseline measurements were obtained, all animals stayed in the supine position and ventilated (TLV) via DLT for 3 hours and 30 minutes.

Haemodynamics and blood gas analyses were monitored at the following time points: at baseline, 15 minutes after OLV/apnoea, 3 hours after OLV/OLF, and after 30 minutes of TLV following deflooding. In the $\mathrm{CO}$ group, measurements were performed temporally in accordance with the OLF group.

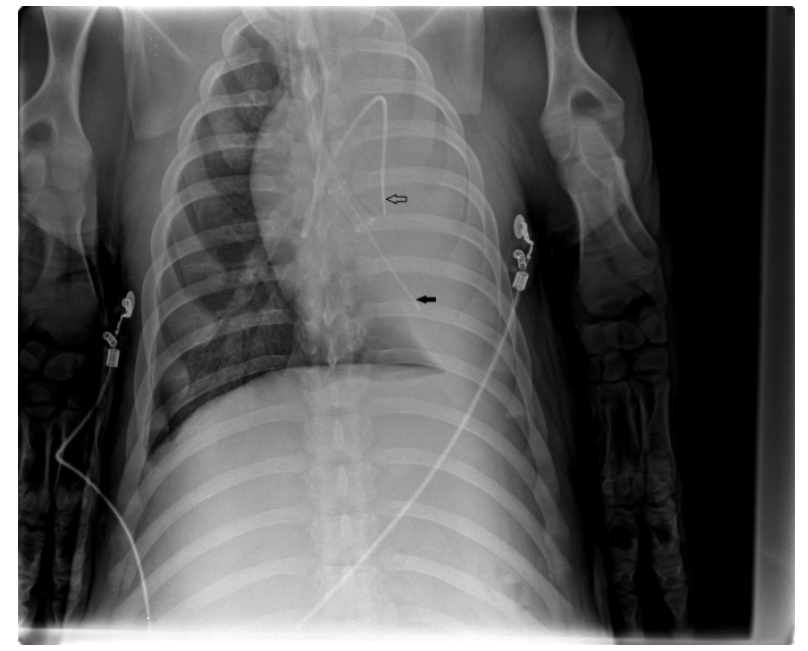

Figure 1. Chest $X$-ray after one-lung flooding of the left lung, showing the pulmonary artery catheter in the left main artery (arrow) and the intrapulmonary catheter via the left bronchial lumen of the DLT (fat arrow).

\section{Lung ultrasound and extravascular lung water (EVLW)}

For sonographic examination, a sonographic system (Flex Focus 800, BK Medical, Arhus, Denmark) with linear $(8870,18-6 \mathrm{MHz})$ and curved arrays (8815, 10-4 MHz) were used. In all animals following OLF, the central pulmonary artery within the flooded lung was located using transthoracic ultrasound, considering the same probe position on the lateral chest wall. The angle adjusted $\left(<60^{\circ}\right)$ Doppler was positioned in the pulmonary artery, and a sample volume was chosen to cover at least one-half of the entire flow width. The flow curves were recorded hourly during flooding. Comparable duplex sonography of the pulmonary arteries in the right nonflooded lung was impossible due to gas in the ventilated lung.

Transthoracic lung sonography was used to determine pleural effusion and lung water content for both lungs at the end of the 30 minutes of TLV following deflooding. To evaluate the lung water content, B-line and pleural line changes were recorded.

Following euthanasia, the thoracic cavity was carefully opened, and the heart and lungs were harvested. The heart was removed so blood could be drained from the lungs. To measure the EVLW, representative tissue samples were taken from the lobes of both lungs and immediately weighed to obtain the wet weight. The samples were weighed again after 24 hours of drying in an oven at $70^{\circ} \mathrm{C}$. The wet-to-dry ratio was calculated as follows: wet/dry $[\%]=\left(\right.$ weight $_{\text {wet }}-$ weight $\left._{\text {dry }}\right) /$ weight $_{\text {wet }} \times 100$. 


\section{Statistical methods}

All data were analysed using MedCalc Statistical Software, version 19.1.7 (MedCalc Software bv, Ostend, Belgium), and distributions were confirmed using the Q-Q plot. Therefore, median values and interquartile ranges are presented. The MannWhitney $U$ test was used to analyse differences between independent groups (i.e., OLF and CO groups). Statistical significance (two sided) was set at a $P$ value less than 0.05 . For analysis of changes between measurement points within each group, the Friedmann test followed by a post hoc test for $P$ values both less than 0.05 and less than 0.01 were performed.

\section{Results}

In total, 14 animals were examined in this study. Five animals served as controls, and nine animals were studied in the OLF group. One animal died of acute cardiac death as a result of difficult intubation prior to OLF. In one animal, a partial fluid run-over occurred due to DLT dislocation. The data from these animals were included from the beginning of the experiment until the last useful measurement. The DLT was placed again under fibrebronchoscopic control and the liquid was sucked off. The situation could be controlled without harm. No animals died due to OLF.

The baseline animal data showed no differences between groups. The systolic and mean pulmonary artery pressures (SPP and MPP) increased marginally in the OLF group compared to the $\mathrm{CO}$ group by 6 $\mathrm{mmHg}$ and $5 \mathrm{mmHg}$, respectively [SPP: 35 (24.3-36.0) mmHg vs. 29 (27.3-31.3) $\mathrm{mmHg}, P=0.004$; MPP: 28 (27.0-28.0) $\mathrm{mmHg}$ vs. 23 (20.8-26.5) $\mathrm{mmHg}, P=0.02]$. Heart rate, mean arterial pressure, and diastolic pulmonary artery pressure were unchanged during the entire study. During OLF, the intrapulmonary pressure in the flooded lung was 13 [9.5-14.5] $\mathrm{mmHg}$ (Table 1).

The $\mathrm{PaO}_{2}\left(\mathrm{FIO}_{2}=0.4\right)$ during OLF was slightly decreased in the OLF group compared to the CO group $\left[\begin{array}{llll}131.8 \quad(110.3-143.0) & \mathrm{mmHg} & \text { vs. } 158.2\end{array}\right.$ (150.5-166.1) $\mathrm{mmHg}, P=0.02]$ and baseline values [vs. 153.3 (139.4-165.6) mmHg, $P<0.01]$. Arterial oxygen saturation $\left(\mathrm{SaO}_{2}\right)$, arterial carbon dioxide partial pressure $\left(\mathrm{PaCO}_{2}\right)$, and $\mathrm{pH}$ were unchanged in the OLF group compared to the $\mathrm{CO}$ group (Fig. 2, 3)).

In the OLF group during OLV/OLF, the pulmonary right-to-left shunt fraction (Qs/Qt) was $4 \%$ higher than in the $\mathrm{CO}$ group, but this difference was not significant. In contrast to OLV/OLF, OLV/ apnoea led to a significant decrease in the partial pressure of oxygen and an increase in the shunt fraction compared to the $\mathrm{CO}$ group $\left[\mathrm{PaO}_{2}\right.$ (1.0): 205.5
(191.2-216.4) $\mathrm{mmHg}$ vs. $461.6(451.7-478.7) \mathrm{mmHg}, P=$ 0.01; Qs/Qt: $0.3(0.28-0.34)$ vs. $0.16(0.12-0.18), P=$ 0.01]. After flooding of the nonventilated lung, $\mathrm{PaO}_{2}$ increased and the shunt fraction decreased significantly compared to OLV/apnoea $\left[\mathrm{PaO}_{2}(1.0)\right.$ : 400.1 (338.1-464.7) vs. 205.5 (191.2-216.4) $\mathrm{mmHg}, P<$ 0.01 ; Qs/Qt: $0.19(0.14-0.23)$ vs. $0.3(0.28-0.34), P<$ 0.01]; Table 2, Fig. 4].

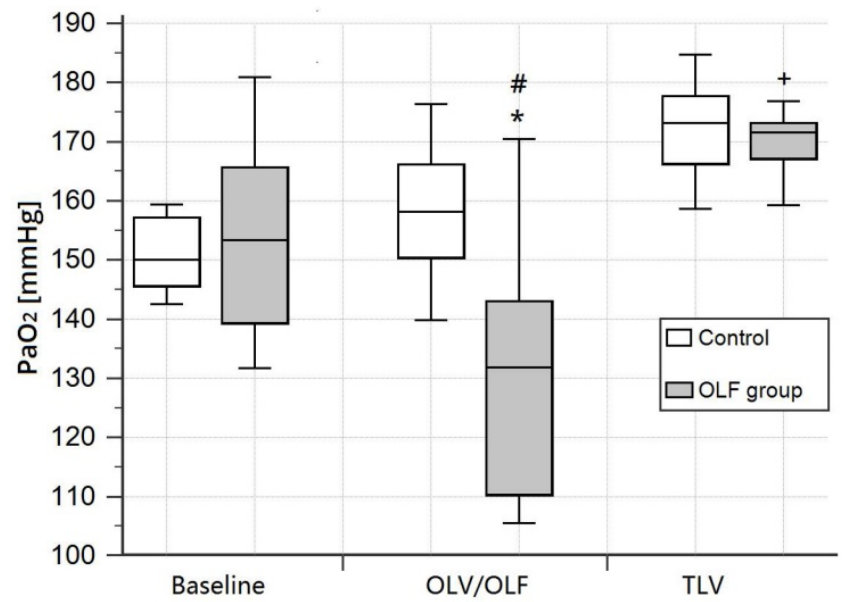

Figure 2. Arterial oxygen partial pressure $\left(\mathrm{PaO}_{2}\right)$ at baseline, during one-lung ventilation/one-lung flooding (OLV/OLF), and after deflooding and two-lung ventilation (TLV). Abbreviations: $\mathrm{PaO}_{2}$ : arterial oxygen partial pressure using an inspired fraction of oxygen of $0.4, \mathrm{OLV}$ : one-lung ventilation, OLF: one-lung flooding, TLV: two-lung ventilation, CO group: control group, OLF group: OLF experimental group. $* P<0.05$, significant compared with the $C O$ group, $\# P<0.01$, significant compared with baseline and TLV values, $+P<0.05$, significant compared with baseline values.

Table 1. Haemodynamics at baseline, during one-lung flooding and after deflooding and two-lung ventilation

\begin{tabular}{|c|c|c|c|c|}
\hline Group & Baseline & OLV/OLF & TLV & $P$ Value \\
\hline \multicolumn{5}{|l|}{ HR, bpm } \\
\hline $\mathrm{CO}$ group & $96(91.5-101.5)$ & 92 (89.3-98.0) & 94 (92.7-97.5) & 0.54 \\
\hline OLF group & 99(94.3-110.7) & 95 (92.5-103.3) & $91^{\#}(87.5-100.5)$ & 0.02 \\
\hline$P$ value* & 0.26 & 0.31 & 0.55 & \\
\hline \multicolumn{5}{|c|}{ MAP, mmHg } \\
\hline CO group & 79 (75.3-86.8) & 75 (70.3-81.3) & $81(59.0-82.8)$ & 0.85 \\
\hline OLF group & $84(80.3-96.0)$ & $79(63.0-89.8)$ & $90(79.8-101.3)$ & 0.09 \\
\hline$P$ value* & 0.21 & 0.69 & 0.12 & \\
\hline \multicolumn{5}{|l|}{$\mathrm{SSP}, \mathrm{mmHg}$} \\
\hline CO group & $29(26.5-30.3)$ & $29(27.3-31.3)$ & $28(26.8-28.5)$ & 0.41 \\
\hline OLF group & $30(29.0-31.5)$ & $35^{\#}(33.5-35.5)$ & $32(27.5-37.0)$ & 0.02 \\
\hline$P$ value* & 0.2 & 0.004 & 0.16 & \\
\hline \multicolumn{5}{|l|}{$\mathrm{DPP}, \mathrm{mmHg}$} \\
\hline CO group & $19(16.8-20.3)$ & $22(18.5-23.3)$ & $19(17.8-21.5)$ & 0.37 \\
\hline OLF group & $21(19.3-21.8)$ & $21(20.0-23.0)$ & $22(19.0-25.0)$ & 0.56 \\
\hline$P$ value* & 0.19 & 0.9 & 0.17 & \\
\hline \multicolumn{5}{|c|}{ MPP, mmHg } \\
\hline CO group & $24(21.8-25.3)$ & $23(20.8-26.5)$ & $24(23.8-25.3)$ & 0.96 \\
\hline OLF group & $25(24.0-25.3)$ & $28+(27.0-28.0)$ & $26^{+}(25.2-29.0)$ & 0.007 \\
\hline$P$ value* & 0.16 & 0.02 & 0.027 & \\
\hline \multicolumn{5}{|l|}{ IPP, mmHg } \\
\hline OLF group & & $13(9.5-14.5)$ & & \\
\hline
\end{tabular}

Abbreviations: HR: heart rate, MAP: mean artery pressure, SPP: systolic pulmonary artery pressure, DPP: diastolic pulmonary artery pressure, MPP: mean pulmonary artery pressure, IPP: intrapulmonary pressure, OLV: one-lung ventilation, OLF: one-lung flooding, TLV: two-lung ventilation, $\mathrm{CO}$ group: control group, OLF group: experimental OLF group;

${ }^{*} P<0.05$, significant compared with the CO group, ${ }^{*} P<0.05$, significant compared with baseline values, ${ }^{+} P<0.01$, significant compared with baseline values. 
Table 2. Arterial oxygen partial pressure and shunt fraction at baseline, during one-lung ventilation/apnoea, one-lung flooding, and after deflooding and two-lung ventilation

\begin{tabular}{|c|c|c|c|c|c|c|}
\hline Characteristic & & Baseline & OLV/apnoea & OLV/OLF & TLV & $P$ Value \\
\hline \multirow[t]{3}{*}{$\mathrm{PaO}_{2}, \mathrm{mmHg}$} & CO group & $474.4(463.4-486.0)$ & $461.6(451.7-478.7)$ & $450(442.4-465.0)$ & $497.8(442.3-515.3)$ & 0.35 \\
\hline & OLF group & $453.9(438.8-494.2)$ & $205.5^{\#}(191.2-216.4)$ & $400.1^{+}(338.1-464.7)$ & $444.2(400.3-470.7)$ & 0.0015 \\
\hline & $P$ value ${ }^{*}$ & 0.61 & 0.01 & 0.22 & 0.22 & \\
\hline \multirow[t]{3}{*}{ Qs/Qt } & $\mathrm{CO}$ group & $0.15(0.14-0.153)$ & $0.16(0.125-0.183)$ & $0.15(0.148-0.16)$ & $0.14(0.13-0.18)$ & 0.6 \\
\hline & OLF group & $0.17(0.16-0.22)$ & $0.3^{\#}(0.28-0.34)$ & $0.19+(0.14-0.23)$ & $0.16(0.13-0.15)$ & 0.0035 \\
\hline & $P$ value ${ }^{*}$ & 0.077 & 0.01 & 0.22 & 0.27 & \\
\hline
\end{tabular}

Abbreviations: $\mathrm{PaO}_{2}$ : arterial oxygen partial pressure using an inspired fraction of oxygen of 1.0, Qs/Qt: right-to-left shunt fraction, OLV: one-lung ventilation, OLF: one-lung flooding, TLV: two-lung ventilation, $\mathrm{CO}$ group: control group, OLF group: OLF experimental group;

${ }^{*} P<0.05$, significant compared with the CO group, ${ }^{\#} P<0.01$, significant compared with baseline values, $+P<0.01$, significant compared with OLV/apnoea.

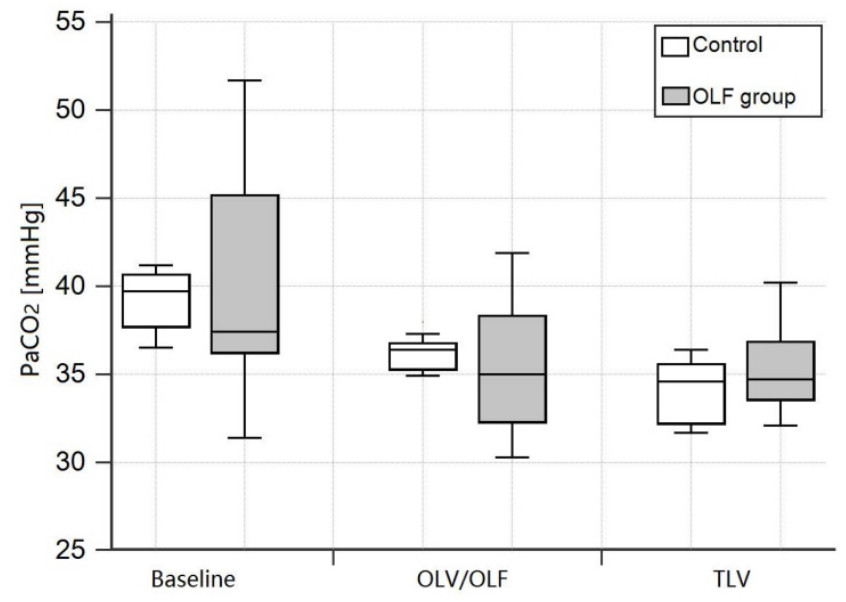

Figure 3. Arterial carbon dioxide partial pressure $\left(\mathrm{PaCO}_{2}\right)$ at baseline, during one-lung ventilation/one-lung flooding (OLV/OLF), and after deflooding and two-lung ventilation (TLV). Abbreviations: $\mathrm{PaCO}_{2}$ : arterial carbon dioxide partial pressure, OLV: one-lung ventilation, OLF: one-lung flooding, TLV: two-lung ventilation, CO group: control group, OLF group: OLF experimental group. Changes in the OLF group are non-significant.

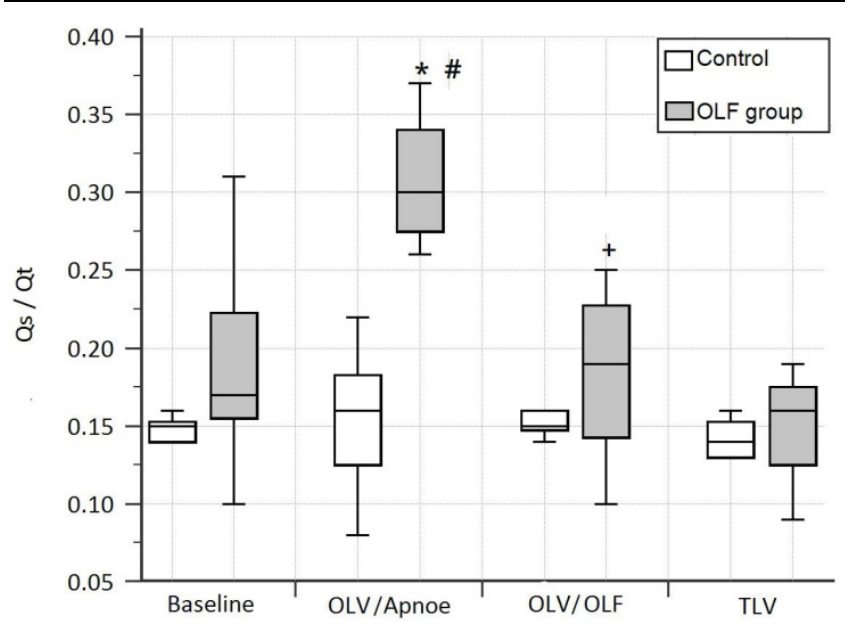

Figure 4. The shunt fraction $(\mathrm{Qs} / \mathrm{Qt})$ at baseline, during one-lung ventilation/apnoea (OLV/apnoea), during one-lung ventilation/one-lung flooding (OLV/OLF), and after deflooding and two-lung ventilation (TLV). OLF experimental group (grey); control group (white). $* P<0.05$, significant compared with the CO group, \#P<0.01, significant compared with baseline values, $+P<0.01$, significant compared with OLV/apnoea.

After deflooding and TLV, haemodynamics and gas exchange indices showed no differences compared to the $\mathrm{CO}$ group and baseline values, respectively.
Color-coded duplex sonography of the pulmonary artery within the flooded lung revealed a strong oscillating flow pattern, which was found in all experiments (Fig. 5).

The amount of liquid recovered was $297.5 \mathrm{~mL}$ (281.8-310.0 mL), which corresponds to a recovery rate of $63.2 \%$ [saline filling amount: $475 \mathrm{~mL}$ (463-496 $\mathrm{mL})]$. Thirty minutes after deflooding and TLV, the EVLW volume was $11 \%$ higher in the flooded lung compared to the CO group [78.9\% (75.5-84.3\%) vs. $70.8 \%(70.3-72.3 \%)]$, but these differences were not significant (Fig. 6).

At this point, lung sonography showed few B-lines and a slight increase of pleura thickening in absence of subpleural consolidations or pleural effusions of the previously flooded lung compared to the right lung (Fig. 7).

\section{Discussion}

In this study, the effects of OLF on haemodynamics and gas exchange with closed thorax were examined. The key result was that OLF did not influence haemodynamics or gas exchange during or after flooding in a clinically relevant manner. OLV and apnoea in the nonventilated lung caused a relevant decrease in $\mathrm{PaO}_{2}$ and an increase of shunt fraction, which are well-known problems during thoracic surgery using OLV without recruitment strategies of the nonventilated lung, such as CPAP or HFJV [7-9]. In contrast, such deterioration of both oxygenation and ventilation did not occur under OLV/OLF. Previous studies showed comparable results under the conditions of thoracoscopy or thoracotomy $[14,15]$. Thus, the conditions under which the OLF is performed (at opened or closed thorax) do not influence the hemodynamics and gas exchange. Saline filling of one lung with half of the functional residual capacity led to an intrapulmonary pressure of $13 \mathrm{mmHg}$, which is twice the capillary pressure. Our previous measurements for OLF after thoracotomy showed that the pulmonary capillary pressure (measured by a catheter in the left atrium) was $7 \mathrm{mmHg}$ [26]. The pulmonary capillary wedge 
pressure measurements in this study were not usable because of a false high pressure caused by the placement of the pulmonary artery catheter in the flooded lung. The transmural pressure difference, which includes a markedly higher intrapulmonary pressure, occludes the venous region of the capillary bed. Therefore, it can be assumed that pulmonary perfusion ceases in a large part of the flooded lung.

Although one-time unilateral lung flooding must be distinguished from bronchopulmonary whole-lung lavage, the physiologic alterations can be anticipated from the hemodynamic response of the pulmonary circulation to the variations in the airway pressure that occur as the airways are cyclically filled and emptied. The highest oxygen saturation level is usually seen at the completion of the filling phase when the blood is physiologically shunted from the nonventilated to the ventilated lung. Conversely the oxygen level will drop as the lavage lung is emptied [16].

The oscillating flow in the pulmonary artery of the flooded lung is supportive evidence of the arrest of the pulmonary circulation. Similarly, oscillating flow patterns recorded in the extracranial cerebral arteries of brain-dead patients are considered to be conclusive signs of cerebral circulatory arrest $[27,28]$. The oscillating Doppler pattern is defined as a biphasic flow velocity spectrum with equivalent, opposing inflow and outflow components, such that the resulting time-averaged mean velocity in the evaluated vessel is zero [29].

The insignificant increase in the shunt fraction $(4 \%)$ in the OLF group compared to the CO group could have different causes. First, both the intrapulmonary pressure and the right-to-left shunt volume are dependent on the filling volume. In previous experiments to determine the correct fill volume, we observed an increased shunt fraction when the fill volume was less than $12.5 \mathrm{~mL} / \mathrm{kg}$ body mass. Second, in the nondependent regions of the flooded lung where the hydrostatic intrapulmonary pressure is below $7 \mathrm{mmHg}$, perfusion still occurs due to uniform blood flow [30]. Third, due to redistribute blood from the flooded into the ventilated lung an increased perfusion/ventilation mismatch in the ventilated lung can occur, causing an increase in the shunt fraction in the ventilated lung.

Despite an approximate $50 \%$ reduction in the pulmonary artery cross-sectional area as a result of the flooding of one lung, both the SPP and MPP increased marginally by $6 \mathrm{mmHg}$ and $5 \mathrm{mmHg}$, respectively, compared to the $\mathrm{CO}$ group. Both vascular compliance and capillary recruitment are mechanisms in the ventilated lung that can compensate for the increased vascular resistance in the flooded lung. Similarly, a considerable increase in cardiac output in healthy humans is accompanied only by a marginal increase in pulmonary arterial pressure [31].

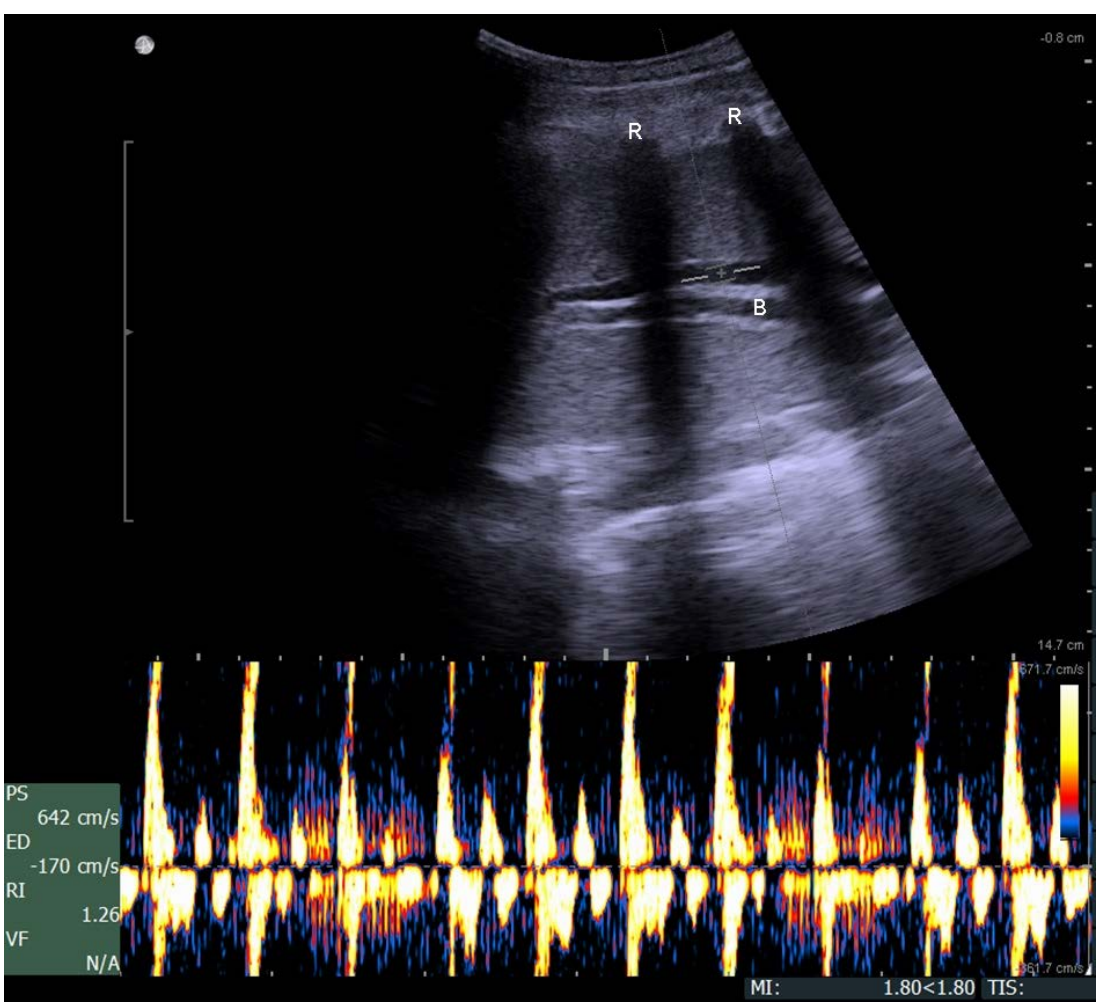

Figure 5. Duplex sonography imaging shows a biphasic oscillating flow in the pulmonary artery within the flooded lung (B, bronchus; R, ribs). 


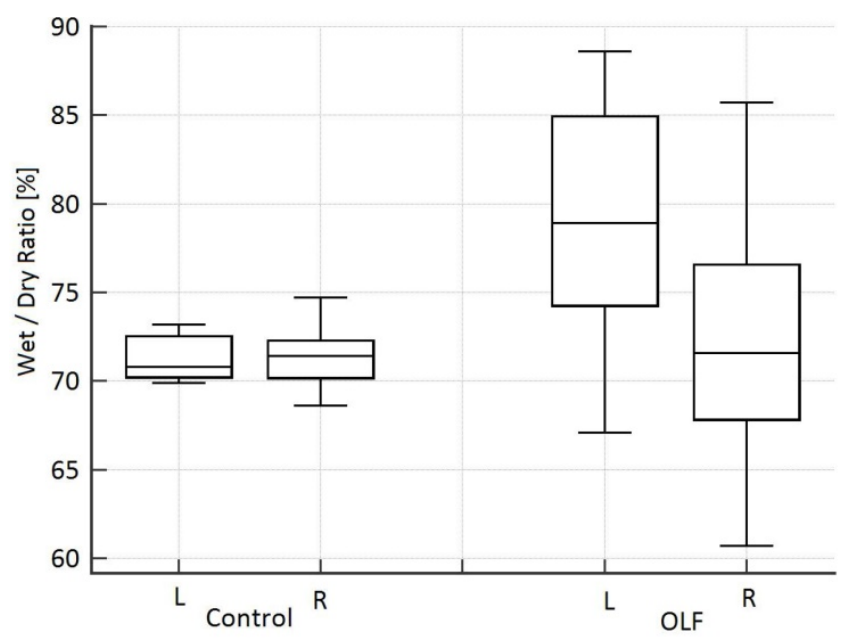

Figure 6. Wet/dry weight ratio in both lungs ( $L=$ left lung, flooded for 3 hours; $R=$ right lung, only ventilated) 30 minutes after deflooding and two-lung ventilation in the one-lung flooding (OLF) group compared to the control group.

After deflooding and TLV over 30 minutes, there is little residual fluid volume in the left lung compared to the right nonflooded lung. The EVLW was $11 \%$ higher than in the right lung. In a previous study we found a $6.2 \%$ higher EVLW in the flooded lung, however the flooding time was only 60 minutes [32]. The described method to determine EVLW cannot differentiate between intraalveolar and interstitial fluid accumulation. But we found a slight increase of B-lines that indicate oedematous thickening of interlobular septa [33]. This residual fluid does not negatively impact the gas exchange. $\mathrm{PaO}_{2}, \mathrm{PaCO}_{2}$, and the shunt fraction 30 minutes after TLV did not differ in the OLF group compared to the $\mathrm{CO}$ group and baseline values.

Our study has some limitations that should be addressed. First, the study was carried out on animals with healthy lungs. OLF in patients with chronic obstructive or interstitial lung disease could affect the haemodynamics and gas exchange indices. However, whole-lung lavage under OLV to treat pulmonary alveolar proteinosis, which causes severe hypoxemic respiratory failure, was considered safe. In one survey of 20 worldwide centres that perform whole-lung lavage in adults, the most common indications were declining lung function, declining oxygenation, and radiographic worsening [34]. The second limitation was the use of the present calculation of the intrapulmonary right-to-left shunt volume because no differentiation can be made between the shunt volume percentages of the right and left lungs. We hypothesize that the $4 \%$ increase in the shunt fraction during OLF/OLV was caused by a ventilation/ perfusion mismatch in the ventilated lung due to redistribute blood from the flooded into the ventilated lung. Only the determination of the side-separated shunt volume would help clarify this hypothesis. In a previous study, we described a novel intraoperative method to determine side-separated shunt volumes [26]. A third limitation was that the investigations were carried out the procedure only with the animals in the supine position. There is a need to examine the haemodynamics and gas exchange indices with the animals in the lateral decubitus position, such as with the flooded lung in both nondependent and dependent positions, because therapeutic procedures, such as ultrasound- or magnetic resonance-guided focused ablation of lung tumours, require various positions. Finally, the study used an acute experimental model to collect data only up to 30 minutes after deflooding and TLV under general anaesthesia. Survival experiments must be performed where gas exchange indices are measured after extubation and spontaneous ventilation to get closer to future clinical practice.
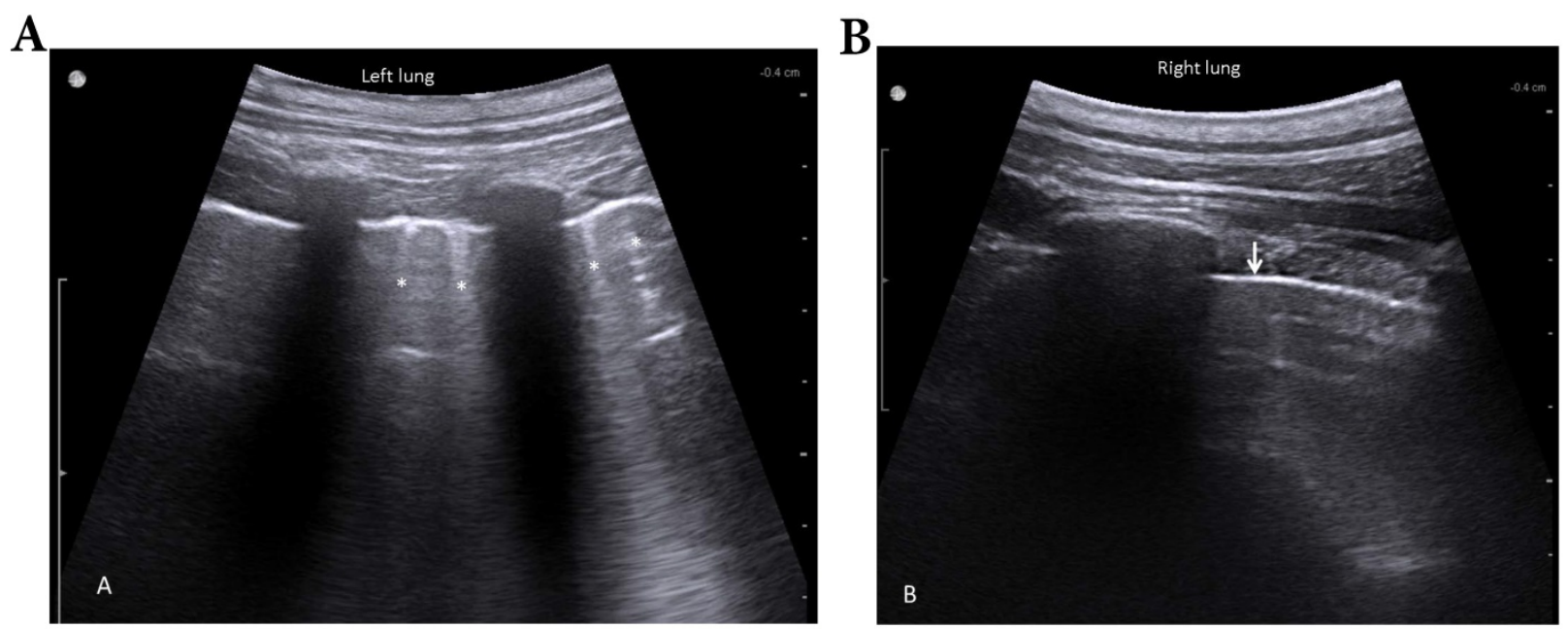

Figure 7. Lung sonography 30 minutes after deflooding and two-lung ventilation. Illustrated are the left lung (filled with saline over 3 hours, A) and the right lung (only ventilated, B). The left lung shows a slightly thickened pleural line and few B-lines (stars); the right lung presents a sharp pleural line (arrow) and no B-lines. 


\section{Conclusions}

We examined whether haemodynamics and gas exchange parameters were influenced by OLV/OLF with closed thorax in a clinically relevant manner. We demonstrated that OLV/OLF influenced the parameters only slightly and maintained them in the normal physiological range. Due to circulatory arrest in the flooded lung, the right-to left shunt volume in the nonventilated lung was minimized. This finding implies that OLF overcomes the declines in oxygenation and ventilation that occur during OLV alone. Additionally, the residual fluid after deflooding and TLV over 30 minutes does not negatively impact the haemodynamics and gas exchange indices. These results should be verified in experiments measuring effects on survival.

\section{Acknowledgments}

The authors thank PD Dr. U. Leder, MBA, A. Peuke and M. Lechner from the SRH Wald-Klinikum Gera for their support and Dipl. Ing. F. Sick, MEDICOPLAST, for providing double lumen endobronchial tubes. We also thank the team of the Central Experimental Animal Facility, University Hospital Jena, especially Mrs. Dobermann, Mrs. Goebel, and Mr. Wuckelt, for their assistance with this project. We thank Dr. Martin Roskos for providing blood gas analyser.

\section{Funding}

The authors gratefully acknowledge the financial support provided by the SRH Wald-Klinikum Gera. The funder had no role in the study design, data collection and analysis, decision to publish, or preparation of the manuscript.

\section{Author Contributions}

- Conceptualization: Thomas Lesser, Reiner Gottschall;

- Formal analysis: Thomas Lesser, Frank Wolfram;

- Funding acquisition: Thomas Lesser, Frank Wolfram;

- Investigation: Thomas Lesser, Reiner Gottschall, Conny Braun;

- Project administration: Thomas Lesser;

- Writing - original draft: Thomas Lesser;

- Writing - review and editing: Reiner Gottschall, Frank Wolfram.

\section{Competing Interests}

The authors have declared that no competing interest exists.

\section{References}

1. Lesser T, Klinzing S, Schubert H, Klein U, Bartel M. Lung flooding--a new method for complete lung sonography. Res Exp Med. 1998; 198: 83-91.

2. Lesser TG, Schubert H, Bischoff S, Wolfram F. Lung flooding enables efficient lung sonography and tumour imaging in human ex vivo and porcine in vivo lung cancer model. Eur J Med Res. 2013; 18: 23-31

3. Wolfram F, Boltze C, Schubert H, Bischoff S, Lesser TG. Effect of lung flooding and high-intensity focused ultrasound on lung tumours: an experimental study in an ex vivo human cancer model and simulated in vivo tumours in pigs. Eur J Med Res. 2014; 19: 1-9

4. Wolfram F, Reichenbach JR, Lesser TG. An ex vivo human lung model for ultrasound-guided high-intensity focused ultrasound therapy using lung flooding. Ultrasound Med Biol. 2014; 40: 496-503.

5. Lesser TG, Petersen I, Pölzing F, Wolfram F. One-Lung Flooding Enables Ultrasound-Guided Transthoracic Needle Biopsy of Pulmonary Nodules with High Sensitivity. Ultrasound Med Biol. 2018; 44: 1556-1562.

6. Kozian A, Schilling T, Fredén F, Fredén F, Maripuu E, Röcken C, Strang C et al. One-lung ventilation induces hyperperfusion and alveolar damage in the ventilated lung: an experimental study. Br J Anaesth . 2008; 100:549-559.

7. Abe K, Mashimo T, Yoshiya I. Arterial oxygenation and shunt fraction during one-lung ventilation: A comparison of isoflurane and sevoflurane. Anesth Analg. 1998; 86: 1266-1270.

8. Kellow NH, Scott AD, White SA, Feneck RO. Comparison of the effects of propofol and isoflurane anaesthesia on right ventricular function and shunt fraction during thoracic surgery. Br J Anaesth. 1995; 75:578-582.

9. Cohen E, Eisenkraft JB, Thys DM, Kirschner PA, Kaplan JA. Oxygenation and hemodynamic changes during one-lung ventilation: effects of CPAP10, PEEP10, and CPAP10/PEEP10. J Cardiothorac Anesth. 1988; 2:34-40.

10. Sentürk M, Layer M, Pembeci K, Toker A, Akpir K, Wiedemann K. A comparison of the effects of $50 \%$ oxygen combined with CPAP to the non-ventilated lung vs. $100 \%$ oxygen on oxygenation during one-lung ventilation. Anasthesiol Intensivmed Notfallmed Schmerzther. 2004; 39:360-364.

11. Hogue CW Jr. Effectiveness of low levels of non-ventilated lung continuous positive airway pressure in improving arterial oxygenation during one-lung ventilation. Anesth Analg. 1994; 79:364-371.

12. Ender J, Brodowsky M, Falk V, et al. High-frequency jet ventilation as an alternative method compared to conventional one-lung ventilation using double-lumen tubes during minimally invasive coronary artery bypass graft surgery. J Cardiothorac Vasc Anesth. 2010; 24:602-607.

13. Abe K, Oka J, Takahashi H, Funatsu T, Fukuda H, Miyamoto Y. Effect of high-frequency jet ventilation on oxygenation during one-lung ventilation in patients undergoing thoracic aneurysm surgery. J Anesth. 2006; 20:1-5.

14. Klinzing S, Lesser T, Schubert H, Bloos F, Klein U, Bartel M. Hemodynamics and gas exchange during experimental one-lung fluid flooding in pigs. Res Exp Med (Berl). 1999; 199:87-94.

15. Klinzing S, Lesser T, Schubert H, Bartel M, Klein U. One-lung flooding for video-assisted thoracoscopic surgery in animal experiments on pigsoxygenation and intrapulmonary shunt. Res Exp Med (Berl). 2000; 199:333-40.

16. Claypool WD, Rogers RM, Matuschak GM. Update on the clinical diagnosis, management, and pathogenesis of pulmonary alveolar proteinosis (phospholipidosis). Chest. 1984; 85:550-8.

17. Shah PL, Hansell D, Lawson PR, et al. Pulmonary alveolar proteinosis: clinical aspects and current concepts on pathogenesis. Thorax. 2000; 55:67-77.

18. Awab A, Khan MS, Youness HA. Whole lung lavage-technical details, challenges and management of complications. J Thorac Dis 2017; 9:1697-1706.

19. Leucht W, Gregor G, Stier H. Einführung in die Versuchstierkunde. Band IV: Das Miniaturschwein, 1. Auflage. Gustav Fischer Verlag, Jena. 1982.

20. Meurens F, Summerfield A, Nauwynck H, Saif L, Gerdts V. The pig: a model for human infectious diseases. Trends Microbiol. 2012; 20:50-57.

21. Swindle MM, Smith AC. Swine in the Laboratory. Surgery, Anesthesia, Imaging and Experimental Techniques, Third Edition. Taylor \& Francis Group, LLC. 2016.

22. Bikou O, Ishikawa K, Fish KM, Zarragoikoetxea I, Hajjar RJ, Aguero J. Modeling Pulmonary Hypertension: A Pig Model of Postcapillary Pulmonary Hypertension. Methods Mol Biol. 2018; 181:367-383.

23. Katalan S, Falach R, Rosner A, Goldvaser M, Brosh-Nissimov T, Dvir A et al. A novel swine model of ricin-induced acute respiratory distress syndrome. Dis Model Mech. 2017; 10:173-183

24. Schwarzkopf K, Schreiber T, Preussler NP, Gaser E, Hüter L, Bauer R et al. Lung perfusion, shunt fraction, and oxygenation during one-lung ventilation in pigs: the effects of desflurane, isoflurane, and propofol. J Cardiothorac Vasc Anesth. 2003; 17:73-75

25. Kwan M, Woo J, Kwok T. The standard oxygen consumption value equivalent to one metabolic equivalent $(3.5 \mathrm{ml} / \mathrm{min} / \mathrm{kg})$ is not appropriate for elderly people. Int J Food Sci Nutr. 2004; 55:179-82. 
26. Lesser $\mathrm{T}$, Schubert $\mathrm{H}, \mathrm{Klinzing} \mathrm{S}$. Determination of the side-separated pulmonary right-to-left shunt volume. J Med Invest. 2008; 55: 44-50.

27. Yoneda S, Nishimoto A, Nukada T, Kuriyama Y, Katsurada K. To-and-fro movement and external escape of carotid arterial blood in brain death cases. A Doppler ultrasonic study. Stroke. 1974; 5:707.

28. Li Y, Liu S, Xun F, Liu Z, Huang X. Use of Transcranial Doppler Ultrasound for Diagnosis of Brain Death in Patients with Severe Cerebral Injury. Med Sci Monit. 2016; 22:1910-5

29. Hassler W, Steinmetz H, Gawlowski J. Transcranial Doppler ultrasonography in raised intracranial pressure and in intracranial circulatory arrest. J Neurosurg. 1988; 68:745-751

30. West JB, Dollery CT, Matthews CME, Zardini P. Distribution of blood flow and ventilation in saline-filled lung. J. Appl. Physiol. 1965; 20:1107-1117.

31. Guyton AC, Jones CE, Coleman TG. Circulatory physiology: Cardiac output and its regulation. Saunders, Philadelphia. 1973.

32. Klinzing S, Lesser T, Schubert H, Bartel M, Klein U. Wet-to-dry ratio of lung tissue and surfactant outwash after one-lung flooding. Res Exp Med (Berl). 2000; 200:27-33.

33. Radzina $\mathrm{M}$, Biederer J. Ultrasonography of the Lung. Fortschr Röntgenstr. 2019; 191: 909-923.

34. Campo I, Luisetti M, Griese M, et al. Whole lung lavage therapy for pulmonary alveolar proteinosis: a global survey of current practices and procedures. Orphanet J Rare Dis. 2016; 11:115. 